\title{
Aplicación de la espectroscopía infrarroja al estudio de cemento aluminoso
}

\author{
LUCÍA FERNÁNDEZ-CARRASCO, Lda. C. Químicas y TOMÁS VÁZQUEZ, Dr. C. Químicas
}

IETCC (CSIC)

Fecha de recepción: 23-XI-95

ESPAÑA

\section{RESUMEN}

Se han estudiado los componentes mineralógicos del cemento aluminoso hidratado a través de la técnica de espectroscopia de absorción infrarroja. Se ha realizado, también con esta técnica, un anál isis de las muestras que se han obtenido de viguetas que fueron fabricadas con hormigón aluminoso.

Se destaca la importancia de esta técnica como complementaria de otras más habitualmente utilizadas en la caracterización de estos compuestos.

\section{SUMMARY}

Mineralogical components of hydrated high alumina cement were st udied by means of infrared absorption spectroscopy. The same technique was also used to analyze the samples obtained from beams that had been made with high alumina concrete.

The article points up the importance of this technique as complementary to others used more habitually in the characterization of these compounds.

\section{INTRODUCCIÓN}

En la determinación de la naturaleza del conglomerante que contiene un hormigón y, en particular, para el estudio de las características del cemento aluminoso hidratado y de las fases que lo componen, se emplean como técnicas que ofrecen una información más interesante, la Difracción de Rayos X(DRX) y el Análisis Térmico (AT) Diferencial y Gravimétrico. Otro tipo de técnicas utilizadas, tales como Microscopía Electrónica, Calorimetría, Difracción de Energía Dispersiva utilizando Radiaciones Sincrotrón, etc. ofrecen resultados específicos de mucho interés pero precisan, en general, una especialización poco frecuente o conllevan unos costos muy elevados.

No obstante, tanto DRX como AT presentan unas limitaciones que dificultan notablemente la obtención de todos los datos necesarios para alcanzar un conocimiento completo de toda la problemática planteada.

El ATD da idea de las transformaciones físico-químicas que se producen en una sustancia determinada y las temperaturas a las cuales esas transformaciones ocurren. La TG/DTG estudia las variaciones de peso que experimenta una sustancia en función de la temperatura. El estudio cualitativo y (sobre todo) cuantitativo del fenómeno de conversión, así como de los procesos de carbonatación del cemento aluminoso se consigue con gran fidelidad por esta técnica.

Posee como limitación más evidente que los termogramas de las muestras de cemento aluminoso presentan, con frecuencia, una superposición de las curvas de los distintos hidratos, lo que genera dificultades de interpretación.

Otros problemas que se han encontrado en la utilización del AT en los estudios de cemento aluminoso son aquéllos derivados de las posibles alteraciones de las muestras durante el propio registro. Se ha señalado (1) que cuando una muestra de cemento se registra sin secar previamente, en el termograma aparecen picos a $300^{\circ} \mathrm{C}$ y $330^{\circ} \mathrm{C}$ que se corresponden con la deshidratación del hidróxido de aluminio (gibsita obayerita) ydel $\mathrm{C}_{3} \mathrm{AH}_{6}$, respectivamente, aunque la muestra inicialmente contuviese única y 
exclusivamente aluminatos hexagonales. Ello es debido a que la presencia de humedad y calor favorece la transformación del hidrato hexagonal a cúbico en el propio crisol del ATD.

La DRX es la técnica más utilizada en el estudio de los hormigones para determinar si están constituidos por cemento aluminoso o por otro conglomerante, así como para determinar la composición mineralógica de las fases presentes y deducir el grado de conversión sufrido por el cemento.

Su limitación viene fijada por ser una técnica que sólo determina compuestos cristalinos. Es decir, no proporciona información suficiente acerca de las fases gel, amorfas o pobremente cristalizadas. Las fases hidratadas del cemento aluminoso en ocasiones son de esta naturaleza, por lo que es necesario acudir a otros medios para complementar los resultados obtenidos.

En este trabajo se ofrece un repaso de la información que puede obtenerse de la espectroscopía infrarroja, como técnica de análisis capaz de complementar de forma eficaz ciertos aspectos que no pueden abordarse con los estudios de cemento aluminoso realizados a través de DRX o de AT.

Se presentan los espectros IR de las fases más importantes en el estudio del cemento aluminoso, con una breve descripción de éstos. Asimismo se dan los espectros IR del cemento aluminoso hidratado "sin convertir", después de la conversión y de su carbonatación. También se dan los espectros de diversos cementos obtenidos a partir de los hormigones de viguetas con distinto grado de alteración.

Los gráficos de las sustancias puras que se presentan proceden, en su mayor parte, de productos sintetizados para el presente estudio y en los casos que así se indica, han sido tomados de la bibliografia.

\section{ESPECTROSCOPÍA INFRARROJA}

La espectroscopía infrarroja aporta un apoyo, en ocasiones decisivo, a la información que se extrae de la difracción de Rayos X y del Análisis Térmico sobre los fenómenos que sufre en su hidratación y carbonatación el cemento aluminoso; sobre todo en función de la capacidad que ofrece el IR para detectar compuestos no cristalinos. No obstante tiene unas limitaciones prácticas que reducen notablemente su efectividad; así, por ejemplo, las fases hidratadas del cemento aluminoso dan unos espectros IR con bandas de absorción generalmente anchas y mal definidas, lo que dificulta la precisión en las medidas de sus frecuencias de absorción y, por consiguiente, una adecuada identificación. Esto se pone de particular relieve con los aluminatos hidratados hexagonales, que son dificilmente distinguibles entre sí. Lo cual puede explicar, en parte, los diferentes valores que se encuentran en la bibliografía y que han sido dados por diversos investigadores (ver Tabla I). Otro motivo de alguna discordancia es el método de preparación de muestras adoptado, dado que distintos métodos dan, en ocasiones, algunas diferencias en los espectros IR correspondientes. El método más empleado es el de "comprimidos de $\mathrm{KBr}$ ". Se basa en la dilución de una pequeña cantidad de muestra, del orden de miligramos, en una proporción muy superior de bromuropotásico (transparentea la radiación infrarroja), y sometido a presión en un molde adecuado para formar un comprimido que posteriormente es registrado en un espectrofotómetro.

Otro método, bastante utilizado, está basado en la dilución de una pequeña proporción de muestra en un medio líquido adecuado, generalmente parafina líquida, con pocas bandas activas al IR, y se realiza el registro espectrográfico entre cristales transparentes a la radiación infrarroja (de $\mathrm{KBr}$, por ejemplo).

Como ventaja evidente de la espectroscopía infrarroja es su capacidad para detectar tanto compuestos cristalinos como no cristalinos. Además, como aspectos concretos de interés, la técnica es de gran sensibilidad para los compuestos carbonatados, aunque esto-paradójicamentepuede llegar a ser un inconveniente cuando, a causa de una inevitable contaminación, las bandas que generan la baja (o muy baja) proporción de compuestos carbonatados puedan llegar a solapar las correspondientes a compuestos mayoritarios, dificultando su discriminación.

En general, la espectroscopía IR identifica aceptablemente la naturaleza de los grupos $\mathrm{OH}$, y las distintas formas en las que el agua puede estar integrada en un compuesto.

Una ventaja adicional no despreciable es su carácter prácticamente no destructivo, dado que para realizar las determinaciones se precisan cantidades muy pequeñas de muestra.

\subsection{Aluminatos cálcicos hidratados hexagonales: $\left(\mathrm{CAH}_{\mathbf{x}}, \mathrm{C}_{2} \mathrm{AH}_{\mathrm{z}}\right)$}

Una parte del agua contenida en los aluminatos cálcicos hidratados hexagonales está ligada con muy poca energía. Con frecuencia las moléculas de agua - $\mathrm{x}, \mathrm{y}, \mathrm{z}-$ unidas a $\mathrm{CAH}_{\mathrm{x}}, \mathrm{C}_{2} \mathrm{AH}_{\mathrm{z}}$ y $\mathrm{C}_{4} \mathrm{AH}_{\mathrm{y}}$, respectivamente toman valores de 10,8 y 13 , pero el contenido de agua de esos compuestos puede disminuir en cantidades discretas sin alterarse de forma significativa la estructura del compuesto. Así por ejemplo, el $\mathrm{CAH}_{10}$ puede perder, sometido a una humedad relativa baja, 3 ó 4,5 moléculas de agua. Los espectros IR de las distintas formas hidratadas del compuesto recogerán esa diferencia principalmente en 
las zonas correspondientes a vibraciones $\mathrm{O}-\mathrm{H}$ (3.000$4.000 \mathrm{~cm}^{-1}$ ) y H-O-H $\left(1.600-1.700 \mathrm{~cm}^{-1}\right)$ (1) (2). Al no alterarse significativamente la estructura de los compuestos, el resto del espectro apenas sufre modificaciones. Esto último, como es lógico, indica recíprocamente, que la variación de unas determinadas proporciones de $\mathrm{H}_{2} \mathrm{O}$ en la composición de los aluminatos hexagonales estudiados no perturba su estructura original.

A este respecto es importante resaltar que el agua más lábil de los aluminatos cálcicos hexagonales tiene una decisiva incidencia en los procesos de transformación del cemento aluminoso (1).

Las asignaciones que se realizan en este trabajo de las bandas de los aluminatos se hace principalmente considerando los modos normales de vibración del grupo de simetría $\mathrm{T}_{d}$ para el $\mathrm{AlO}_{4}$ tetraédrico y del grupo $\mathrm{O}_{\mathrm{h}}$ para el $\mathrm{AlO}_{6}$ octaédrico (3). La interpretación de los espectros IR de los aluminatos cálcicos hidratados ofrecen una dificultad especial, por la forma de las bandas, anchas y mal definidas, que impide asignar sus frecuencias más características con la precisión adecuada.

\subsection{1. $C A H_{10}$ (Fig.1)}

El espectro presenta una banda muy intensa y ancha, característica de vibraciones de valencia $\mathrm{OH}$ en la región de $3.400-3.500 \mathrm{~cm}^{-1}$, con su máximo de absorción hacia $3.500 \mathrm{~cm}^{-1}$. Su asimetría indica que los $\mathrm{OH}$ responsables de la absorción son diferenciados entre sí, al solapar bandas próximas. En $1.650 \mathrm{~cm}^{-1}$ aparece una banda débil característica de vibraciones de deformación $\mathrm{H}-\mathrm{O}-\mathrm{H}$. La absorción en $1.400-1.500 \mathrm{~cm}^{-1}$ es debida a carbonatos producidos por contaminación del $\mathrm{CO}_{2}$ atmosférico.

La zona del espectro correspondiente a la región 1.200$400 \mathrm{~cm}^{-1}$ presenta bandas de muy pobre definición, a causa de vibraciones complejas asociadas y que en este caso puede indicar poca cristalinidad. En todo caso se podrían diferenciar bandas en 1.024 , en 774 (hombro), en 699 y un doblete en $573-528 \mathrm{~cm}^{-1}$.

\subsection{2. $\mathrm{C}_{2} \mathrm{AH} \mathrm{H}_{8}$ (Fig. 1)}

El $C_{2} \mathrm{AH}_{8}$ descrito a continuación es la forma $\beta$, se trata de una modificación polimórfica del aluminato bicálcico hidratado, y se caracteriza por tener su línea de difracción más intensa con un espaciado basal de $10,4 \AA$ (4).

La fórmula de este compuesto puede representarse como sigue:

$$
\mathrm{Ca}_{2}\left[\mathrm{Al}(\mathrm{OH})_{5}\right]_{2} \cdot 3 \mathrm{H}_{2} \mathrm{O}
$$

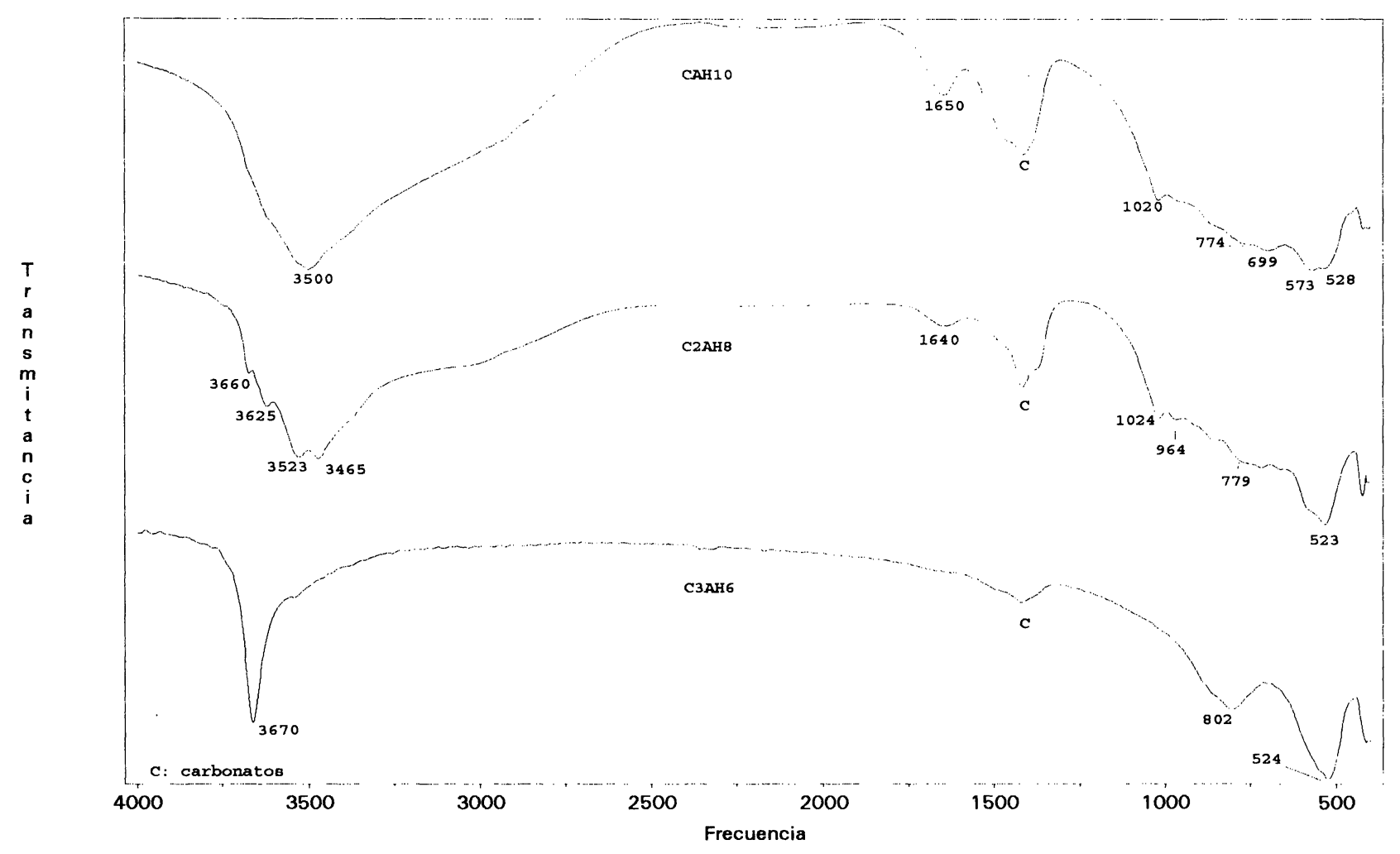

Fig. 1.- Espectros IR de $\mathrm{C}_{3} \mathrm{AH}_{6}, \mathrm{C}_{2} \mathrm{AH}_{8}$ y $\mathrm{CAH}_{10}$. 
Es de destacar las diferencias que se dan en la bibliografia (2)(5) en los espectros IR de este aluminato. El que se ha obtenido en dicho trabajo se encuentra impurificado con una pequeña proporción de carboaluminatos, por contaminación de la muestra con $\mathrm{CO}_{2}$, y de $\mathrm{AH}_{3}$ en forma de gel procedente de la hidratación del aluminato monocálcico. No obstante se incluye por considerar que se encuentran destacadas las bandas más características $\operatorname{del~} \mathrm{C}_{2} \mathrm{AH}_{8}$

En la Tabla I se resumen las frecuencias de absorción caracteristicas de estos aluminatos cálcicos hidratados.
En la zona de $3.400-3.700 \mathrm{~cm}^{-1}$ hay absorciones intensas. Destaca una banda muy fuerte en $3.465 \mathrm{~cm}^{-1} \mathrm{y}$ otra de menor intensidad a $3.625 \mathrm{~cm}^{-1}$ de vibraciones $\mathrm{OH}$, del agua molecular. La forma del espectro es similar al dado en (5), mientras que los valores de las frecuencias presentan alguna diferencia. Los dos hombros en 3.665 y 3.623 $\mathrm{cm}^{-1}$, los interpretamos como debidos a los grupos $\mathrm{OH}$ estructurales. Debe, no obstante, tomarse con cierta precaución estas asignaciones, ya que hay una pequeña proporción de carboaluminatos cálcicos hidratados que pueden, en parte, desvirtuar el espectro. Así, la absorción en 1.350-1.500 $\mathrm{cm}^{-1}$ es debida a vibraciones del

TABLA I

\begin{tabular}{|c|c|c|}
\hline Referencias & $\mathrm{CAH}_{10}$ & $\mathrm{C}_{2} \mathrm{AH}_{8}$ \\
\hline (2) & $\begin{array}{l}3.460-3.380 \\
\text { Entre } 1.100 \text { y } 850 \\
\text { absorción bastante } \\
\text { intensa sin bandas } \\
\text { aisladas. } \\
556 \text { y absorción } \\
\text { entre ese valor y } \\
520 \text {. } \\
420 \text { (poco intensa) }\end{array}$ & $\begin{array}{l}3.465 \\
\text { Entre } 1.100 \text { y } 850 \\
\text { absorción bastante } \\
\text { intensa sin bandas aisladas. } \\
\text { Entre } 535 \text { y } 520 \\
\text { absorción } \\
\text { asimétrica (bandas } \\
\text { solapadas). } \\
530-420\end{array}$ \\
\hline (9) & $\begin{array}{l}3.680(\mathrm{~h})-3.600(\mathrm{~m}) \\
3.540(\mathrm{~h})-3.520(\mathrm{f}) \\
3.475(\mathrm{mf})-1.600(\mathrm{~m}) \\
1.145(\mathrm{~h})-1.115(\mathrm{~h}) \\
1.020(\mathrm{~m})-970(\mathrm{~m}) \\
775(\mathrm{md})-730(\mathrm{~m})- \\
670(\mathrm{~m})-590(\mathrm{~h})- \\
565(\mathrm{~h})-535(\mathrm{f})- \\
425(\mathrm{~m})-415(\mathrm{~m})\end{array}$ & $\begin{array}{l}3.670(\mathrm{f}) \\
3.540(\mathrm{f}) \\
3.495(\mathrm{~h}) \\
1.650(\mathrm{~d}) \\
1.015(\mathrm{~h}) \\
910(\mathrm{~m}) \\
740(\mathrm{f}) \\
595(\mathrm{~h})-535(\mathrm{f}) \\
420(\mathrm{~m})\end{array}$ \\
\hline Este trabajo & $\begin{array}{l}3.500 \text { (mf. Asimétrica; } \\
\text { solapa otras en } \\
\text { mayor y menor } \\
\text { frecuencia) } \\
1.650(\mathrm{~d}) \\
1.020(\mathrm{~m}) \text {. Absorción } \\
\text { continua y difusa } \\
\text { hasta } 800 . \\
774(\mathrm{~h}) \\
699(\mathrm{f} \text {, ancha) } \\
573-528(\mathrm{f} \text {, doblete) }\end{array}$ & $\begin{array}{l}3.660(\mathrm{~h})-3.625(\mathrm{~h}) \\
3.523-3.465(\mathrm{mf}, \\
\text { doblete) } \\
1.640 \\
1.024(\mathrm{f}) \\
964(\mathrm{~m}) \\
779(\mathrm{f}, \text { ancha) } \\
523(\mathrm{mf}, \text { ancha) }\end{array}$ \\
\hline
\end{tabular}

$f=$ fuerte; $m=$ media; $d=$ débil; $h=$ "hombro". 
$\mathrm{CO}_{3}$ de esa impureza. La región del espectro comprendida entre $1.100 \mathrm{y} 400 \mathrm{~cm}^{-1}$ presenta bandas en zonas similares a las dadas para el $\mathrm{CAH}_{10}$ y también de difícil definición.

\subsection{Aluminato cálcico hidratado cúbico: $\mathbf{C}_{3} \mathbf{A H}_{6}$ (Fig.1)}

$\mathrm{El}_{3} \mathrm{AH}_{6}$ es uno de los aluminatos cálcicos hidratados más estudiados. Cristaliza en el sistema cúbico y es el único aluminato cálcico hidratado estable, junto con hidróxido de aluminio e hidróxido cálcico, a temperatura ordinaria, en el sistema $\mathrm{CaO}-\mathrm{Al}_{2} \mathrm{O}_{3}-\mathrm{H}_{2} \mathrm{O}$. Su fórmula puede representarse como sigue:

$$
\mathrm{Ca}_{3}\left[\mathrm{Al}(\mathrm{OH})_{6}\right]_{2}
$$

Su estructura puede describirse como redes formadas por aniones $\left[\mathrm{Al}(\mathrm{OH})_{6}\right]^{3-}$ octaédricos que están unidos entre si por cationes $\mathrm{Ca}^{2+}$.

El espectro IR del $\mathrm{C}_{3} \mathrm{AH}_{6}$ presenta una banda aguda e intensa en $3.670 \mathrm{~cm}^{-1}$ debida a grupos $\mathrm{OH}$ libres. Esta banda aguda es característica del aluminato tricálcico hexahidratado, este hecho posibilita que la técnica de IR sea de gran sensibilidad para la detección de este compuesto. Incluso, la intensidad, la forma aguda de la banda y el estar prácticamente aislada (no solapada por otras bandas intensas) hacen idónea esta técnica para determinar cuantitativamente el contenido de $\mathrm{C}_{3} \mathrm{AH}_{6}$ en una muestra.

En la zona comprendida entre $3.400-3.600 \mathrm{~cm}^{-1}$ se observa una banda ancha, muy débil, que probablemente es causada por agua adsorbida. Prácticamente no se producen absorciones en 1.600-1.700 $\mathrm{cm}^{-1}$ (zona de deformación $\mathrm{H}-\mathrm{O}-\mathrm{H}$ ). Ello indica la no existencia de agua molecular en la estructura del $\mathrm{C}_{3} \mathrm{AH}_{6}$.

Hay una banda en $802 \mathrm{~cm}^{-1}$ y en la zona de $400-700$ $\mathrm{cm}^{-1}$ presenta dos bandas características en 524 y 412 $\mathrm{cm}^{-1}$, respectivamente. No se observan bandas de absorción dignas de mención en el resto del espectro.

Al contrario de lo que sucede con los aluminatos cálcicos hidratados hexagonales, el agua que forma parte de la estructura del $\mathrm{C}_{3} \mathrm{AH}_{6}$ no puede eliminarse parcialmente sin que el conjunto de la estructura, y por consiguiente su espectro IR, sufra una profunda alteración.

\subsection{Hidróxidos de aluminio: (gibsita, bayerita y nordstrandita) (Figs. 2 y 3 )}

\subsubsection{Aspectos generales}

Uno de los temas de mayor interés en el conocimiento de la alteración de viguetas de hormigón de cemento aluminoso es la diferenciación en la polimorfia del $\mathrm{Al}(\mathrm{OH})_{3}$ que pudiera contener. En la actualidad aún no están suficientemente aclarados los parámetros que condicionan la formación de una u otra forma de $\mathrm{Al}(\mathrm{OH})_{3}$ en cementos aluminosos y, por ello, tampoco las razones que justifican la presencia de una $u$ otra forma cristalina determinada en viguetas deterioradas.

Para comprender los fenómenos que afectan a la evolución del cemento aluminoso y por consiguiente de los hidróxidos de aluminio como productos resultantes de la mayor parte de los procesos que puede sufrir el conglomerante, se van a exponer brevemente algunos aspectos de interés relativos a estos compuestos.

La forma cristalina habitual del $\mathrm{Al}(\mathrm{OH})_{3}$ procedente de la hidratación y conversión del cemento aluminoso es la de gibsita. Se ha indicado (6) que un ambiente ácido favorece la cristalización de gibsita, uno neutrode nordstrandita y uno básico de bayerita. Es decir, un $\mathrm{pH}$ elevado de la disolución favorecería más la formación de bayerita que la formación de gibsita. Por otra parte hay una gran diferencia en la velocidad de precipitación entre ambas especies cristalinas: la precipitación lenta favorece la formación de la estructura de gibsita y la rápida la de bayerita (7). Dado que la presencia de álcalis en disolución aumenta la velocidad de las reacciones de hidratación del cemento aluminoso (8) se puede razonablemente inducir que todo ello haya llevado a algunos especialistas a afirmar que la aparición de bayerita indica que los hormigones correspondientes han estado sometidos a alguna acción de los álcalis. $\mathrm{Y}$ ello se ha relacionado con la llamada "hidrólisis alcalina".

No obstante ha de tenerse en cuenta que la bayerita, al envejecer, se transforma en gibsita. Este hecho puede explicar que normalmente sea la gibsita el hidróxido de aluminio que se encuentra en la pasta de cemento aluminoso.

La diferenciación entre bayerita, gibsita y norstrandita se realiza habitualmente mediante DRX. En muchos casos las reflexiones correspondientes más características pueden encontrarse solapadas.

A través de AT también puede existir una difícil discriminación de los picos característicos de las distintas formas del $\mathrm{Al}(\mathrm{OH})_{3}$, interfiriendo, además, con otros hidratos presentes en la muestra.

\subsubsection{Espectroscopía IR}

Aunque las diferencias en la fuerza de unión de los grupos $\mathrm{OH}$ están reflejados principalmente por los numerosos 
máximos de absorción de la zona de $3.000-3.700 \mathrm{~cm}^{-1}$ de las distintas polimorfías del hidróxido de aluminio (9) (Fig.2), la espectroscopía del cemento aluminoso no precisa con claridad la diferencia entre las tres formas del $\mathrm{Al}(\mathrm{OH})_{3}$. No obstante y como orientación, que puede ayudar en la interpretación, se indica que la bayerita tiene una banda en $3.550 \mathrm{~cm}^{-1}$, un hombro en 3.430 y otro hombro en 3.660 (poco precisos, muy extendidos) que no se observan en el espectro de la gibsita.

En la Tabla II se muestra una relación de las bandas de mayor interés de las tres polimorfias, de acuerdo con algunos autores. Puede observarse la no coincidencia de los valores propuestos.

El espectro de la gibsita, sintetizado para este trabajo y que aparece en la Fig.3, tiene la banda en 3.526 que es más intensa que el de la bayerita y, además, el "hombro" en la zona de 3.664 aparece a una frecuencia algo más alta que en el caso de la bayerita. También en $3.377 \mathrm{~cm}^{-1}$ tiene un hombro muy difuso.

\subsection{Carbonato cálcico: (aragonito, calcita, vaterita) y monocarboaluminato de calcio:}

$\left(\mathrm{C}_{3} \mathrm{~A} \cdot \mathrm{CaCO}_{3} \cdot 11 \mathrm{H}_{2} \mathrm{O}\right)$ (Figs. 4 y 5)

Como se ha dicho, la espectroscopía infrarroja es muy sensible para detectar la presencia de carbonatos. En la fig. 4 se muestran los espectros de las tres formas del carbonato de calcio. Las bandas más características y más válidas para su identificación son las que se indican en la Tabla III.

En el trabajo se incluye el espectro İR (Fig.5) de una muestra de un polvo blanco que se formó en la superficie de pasta de cemento aluminoso hidratado a $40^{\circ} \mathrm{C}$ y que posteriormente fue expuesto a la acción del $\mathrm{CO}_{2}$ durante un mes. Una vez analizado por DRX se comprobó que se trataba de monocarboaluminato cálcico hidratado.

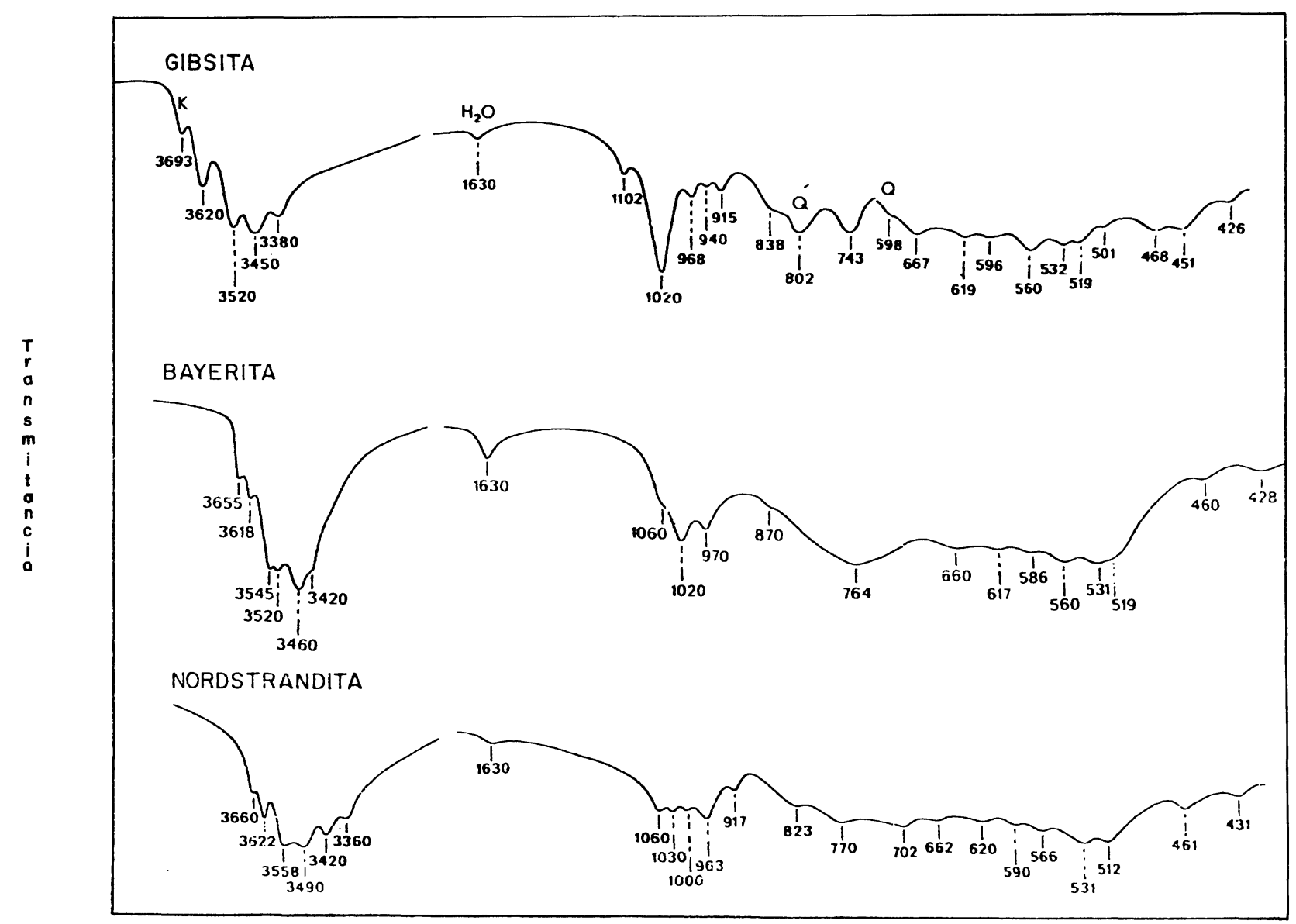

Frecuencia

Fig. 2.- Lispectros IR de la nordstrandita, bayerita y gibsita. 
TABLA II

\begin{tabular}{|c|c|c|}
\hline & $\begin{array}{l}\text { Frecuencias } \\
\text { características }\end{array}$ & Referencias \\
\hline \multirow[t]{4}{*}{ Gibsita } & $\begin{array}{l}3.620-3.521-3.455-3.380-1.020-668-560-450 \\
\text { (intensas) }\end{array}$ & (9) \\
\hline & $3.361-3.378-3.428-3.518-3.616-1.020-967$ & (10) \\
\hline & $3.685-3.613-3.520-3.445-3.390-3.372$ & $(11)^{*}$ \\
\hline & $\begin{array}{l}3.620 \text { (aguda)- } 3.524 \text { y } 3.468 \text { (doblete, muy inten- } \\
\text { sas y anchas)- } 3.395 \text { ("hombro")- } 1.025 \text { (fuerte)- } \\
969 \text { (débil) }\end{array}$ & Este trabajo \\
\hline \multirow[t]{2}{*}{ Bayerita } & $\begin{array}{l}\text { Igual que gibsita, y además en 3.660-1.060-764y } \\
460\end{array}$ & (9) \\
\hline & $\begin{array}{l}3.660-3.620-3.540-3.420-3.401-3.454-3.518- \\
3.533-1.024-975\end{array}$ & (10) \\
\hline Nordstrandita & $\begin{array}{l}3.660-3.558-3.521-3.490-3.455-3.380-3.360- \\
1.060-1.030-823-770-461\end{array}$ & (9) \\
\hline
\end{tabular}

* Sólo se reproducen las vibraciones $\mathrm{OH}$. La banda en $3.685 \mathrm{~cm}^{-1}$ probablemente sea de impureza (¿caolinita?).

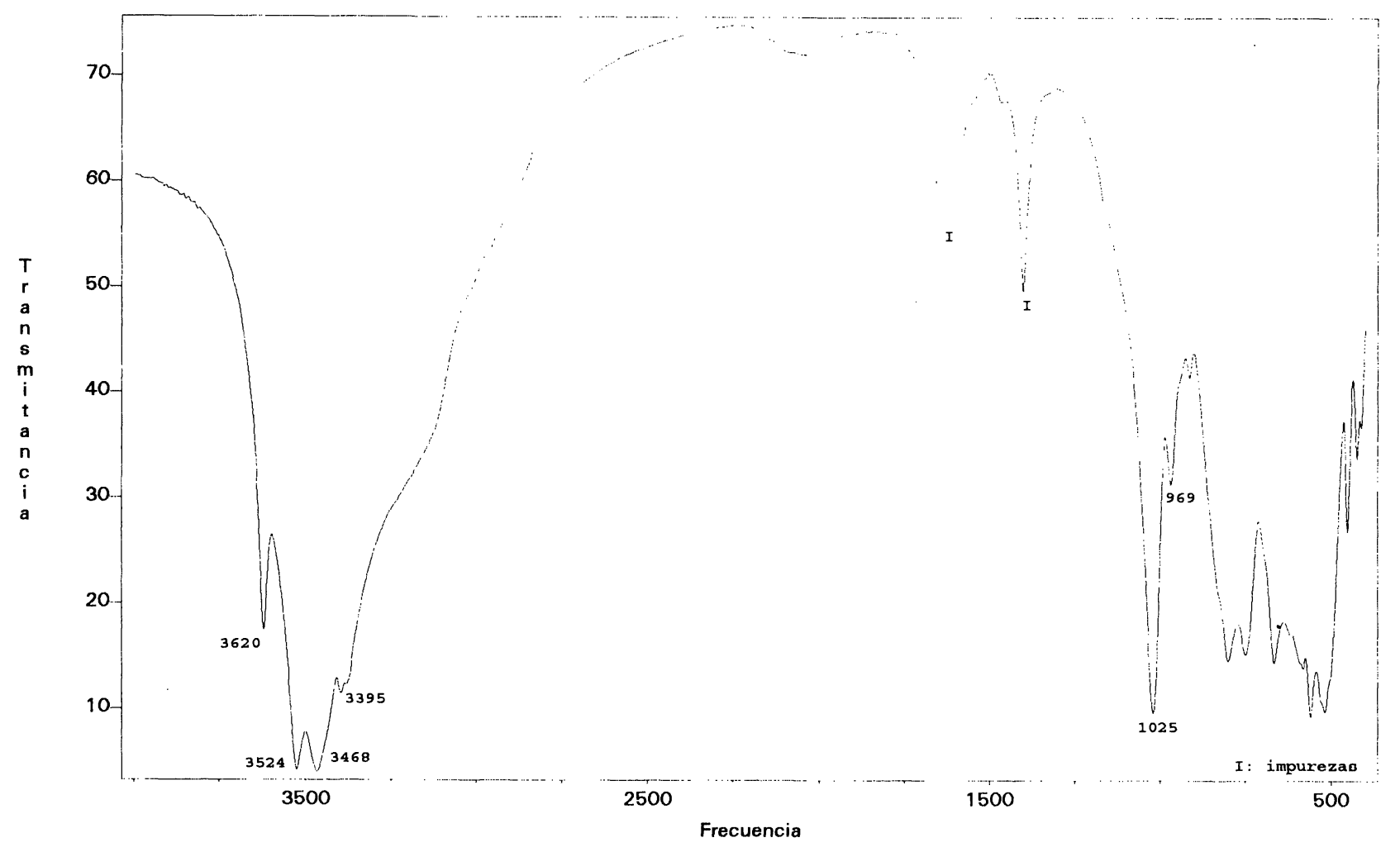

Fig. 3.- Espectro IR de una gibsita sintćtica. 
El espectro presenta en la zona de valencia OH (2.800$3.800 \mathrm{~cm}^{-1}$ aprox.) una absorción ancha muy fuerte $\mathrm{y}$ varias bandas agudas bien definidas. En la Tabla III se muestran las frecuencias de vibración más significativas.
A efectos prácticos, las bandas que se utilizan para su identificación son la de $1.370 \mathrm{~cm}^{-1}$ (aprox.) y la de $420 \mathrm{~cm}^{-1}$

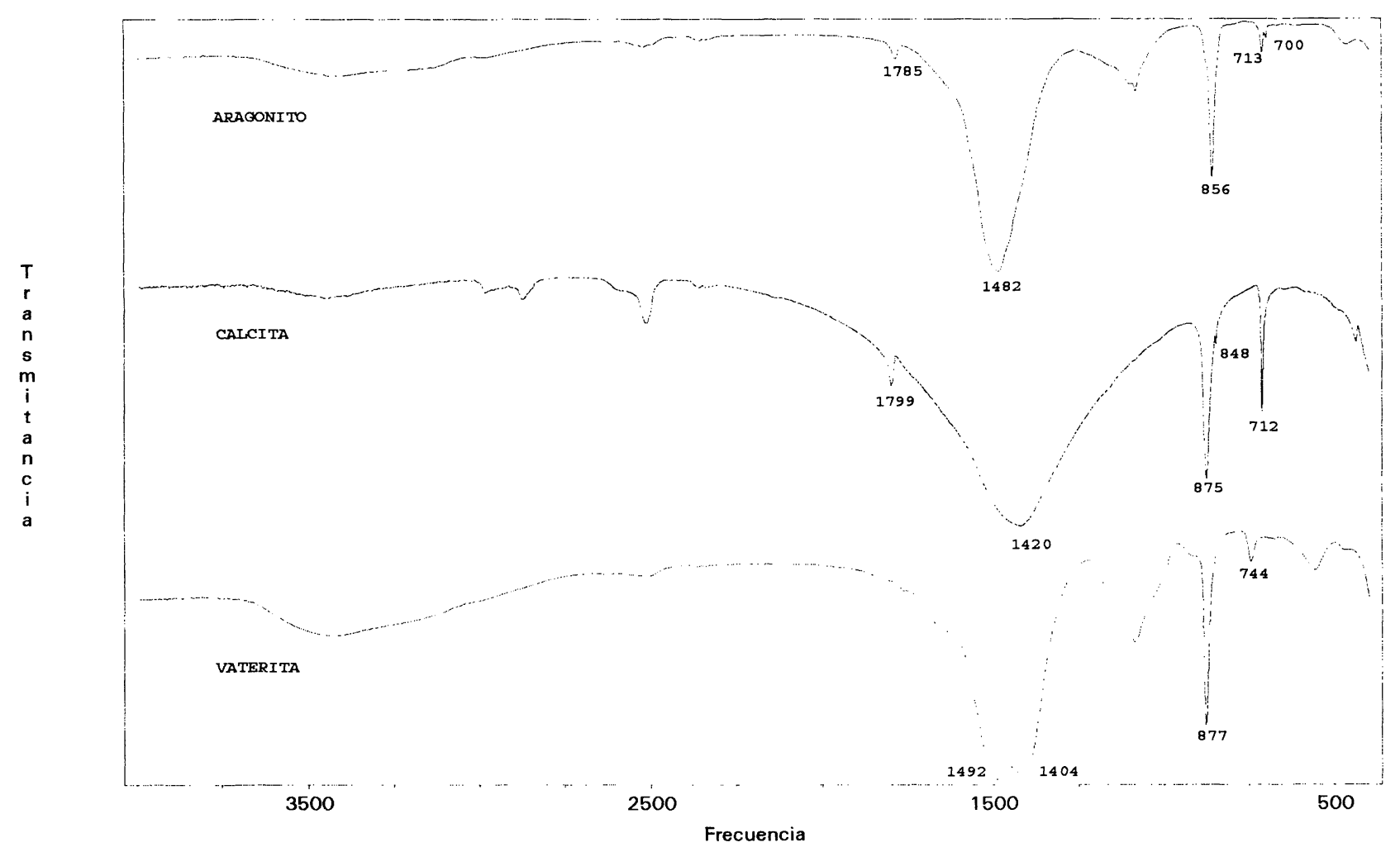

Fig. 4.- Fspectro IR de la vaterita, calcita y aragonito.

TABLA III

\begin{tabular}{|c|c|c|c|}
\hline CALCITA & ARAGONITO & VATERITA & $\mathrm{C}_{3} \mathrm{~A} \cdot \mathrm{CaCO}_{3} \cdot \mathrm{H}_{11}$ \\
\hline $\begin{array}{l}1.799 \text { (md) } \\
1.420 \text { (mf) } \\
875 \text { (m-aguda) } \\
848 \text { (md) } \\
712 \text { (d) }\end{array}$ & $\begin{array}{l}1.785 \text { (md) } \\
1.482 \text { (mf) } \\
856 \text { (m-aguda) } \\
713 \text { (md) } \\
700 \text { (md) }\end{array}$ & $\begin{array}{l}1.492 \\
1.404 \text { (doblete) } \\
\text { (ambas m.f.) } \\
877 \text { (m-aguda) } \\
744 \text { (d) }\end{array}$ & $\begin{array}{l}3.680-3.622 \\
\text { (agudas-f) } \\
3.550-3.523 \\
\text { (solapadas- } \\
\text { anchas-mf) } \\
3.369 \text { (f) } \\
3.130 \text { (h) } \\
3.007 \text { (a-d) } \\
1.641 \text { (d) } \\
1.485^{*}-1.420^{*}- \\
1.369 \text { (mf-a- } \\
\text { solapadas) } \\
953 \text { (f) } \\
877^{\prime \prime}-859^{*} \text { (f-ag) } \\
812-750-719 \\
\text { (débiles) } \\
671 \text { (m) } \\
547 \text { (mf) } \\
424 \text { (f-aguda) }\end{array}$ \\
\hline
\end{tabular}

* Posiblemente debidas a impurificación de $\mathrm{CaCO}_{3}$. 


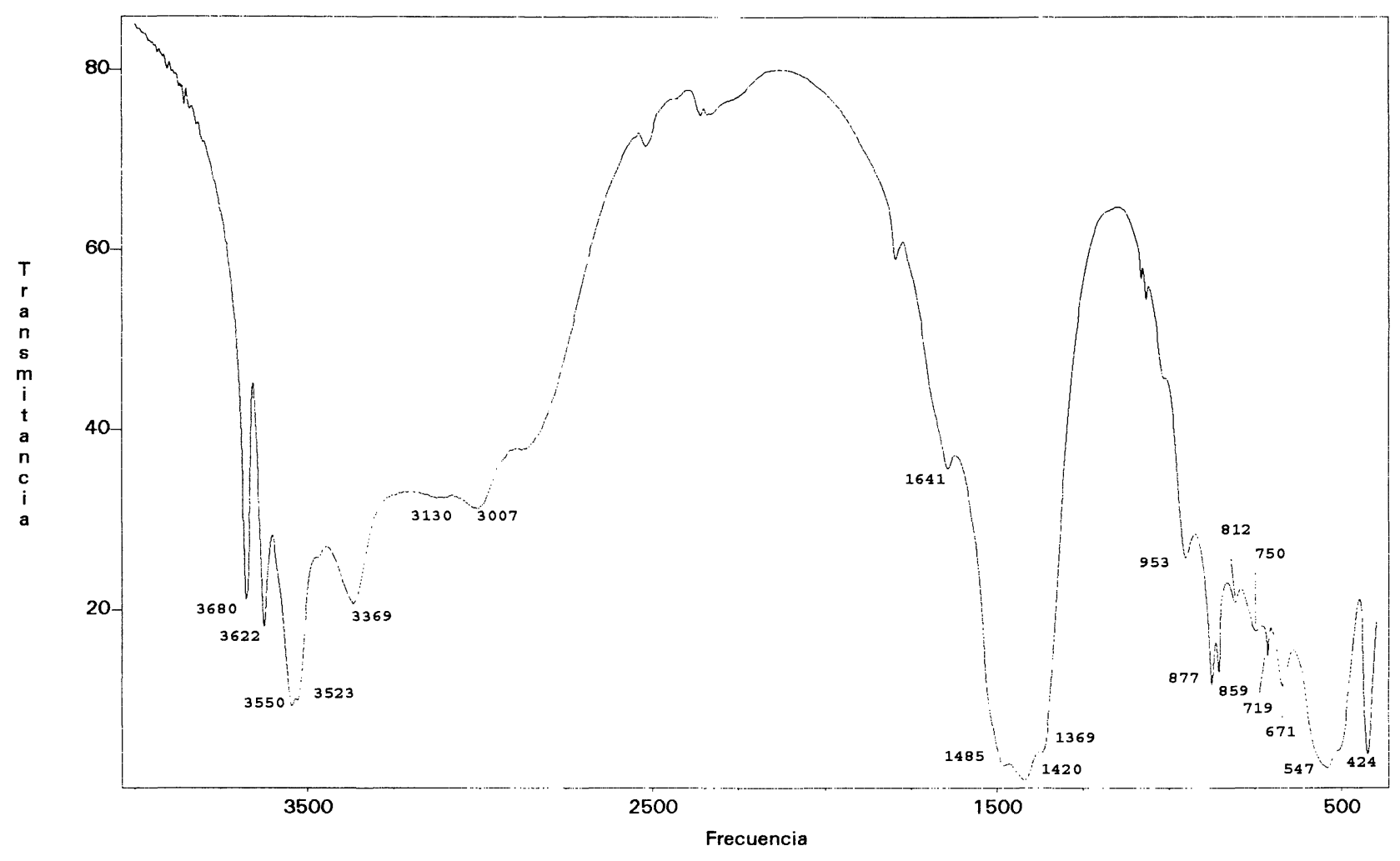

Fig. 5.- Espectro IR de un carboaluminato sintetizado.

El espectro que aquí se presenta coincide esencialmente con el dado por VOLANT (2). Este autor indica que del espectro obtenido puede interpretarse que el monocarboaluminato tiene ocho moléculas de agua combinadas químicamente. No obstante, VOLANT hace notar que no es suficiente la distinción pura y simple entre agua químicamente combinada y agua molecular, ya que es necesario tener en cuenta las interacciones entre los hidroxilos y las moléculas de agua, y además pueden hacerse subdivisiones, dado que el papel del agua puede ser diferente, de acuerdo con las posiciones que ocupen dentro de la estructura de la molécula.

\section{ALGUNOS EJEMPLOS DE ESPECTROS IR DE CEMEN'TO ALUMINOSO. (Figs.6,7,8 y 9)}

Se exponen diversos espectros de cemento aluminoso:

- cemento aluminoso anhidro.

- hidratado a distintas temperaturas en el laboratorio.

- extraído de hormigón de viguetas reales.

\subsection{Cemento aluminoso anhidro}

Se puede destacar la similitud del espectro del cemento aluminoso anhidro con el del aluminato monocálcico (Fig.6), compuesto mayoritario del conglomerante.
Hay algunas diferencias entre los espectros de las sustancias puras y los correspondientes de los componentes del cemento aluminoso, generalmente impuros e influidos por elementos minoritarios y con interacciones mutuas. Además, otros componentes del cementoaluminoso, como la fase ferrítica que también presenta tensiones $\mathrm{Al}-\mathrm{O}$, contribuyen a estas diferencias. Ello hace que las frecuencias características de algunas de las fases aparezcan con ligeras modificaciones con respecto a las teóricas.

\subsection{Cemento aluminoso hidratado en laboratorio}

En laFig. 7 se muestran los espectros del cementoaluminoso hidratado a baja y a elevada temperatura, ambas muestras se hidrataron durante 7 días.

Se observa que con la hidratación los mayores cambios se producen en la zona de $3.000-3.800 \mathrm{~cm}^{-1}$ correspondiente a vibraciones $\mathrm{OH}$. Como es lógico, el espectro del cemento anhidro no tiene bandas en esa zona.

- El espectro de la muestra hidratada a baja temperatura da una banda asimétrica y muy intensa en $3.500 \mathrm{~cm}^{-1}$. Corresponde a vibraciones $\mathrm{OH}$ caracteristicas de aluminatos hexagonales, sobre todo $\mathrm{CAH}_{10}$. Se observa hacia $1.650 \mathrm{~cm}^{-1}$ la banda ancha, debida a vibraciones de deformación H-O-H. Hay bandas en la zona de 1.3501.400 causadas por $\mathrm{CO}_{3}{ }^{2-}$ probablemente de carboaluminato 


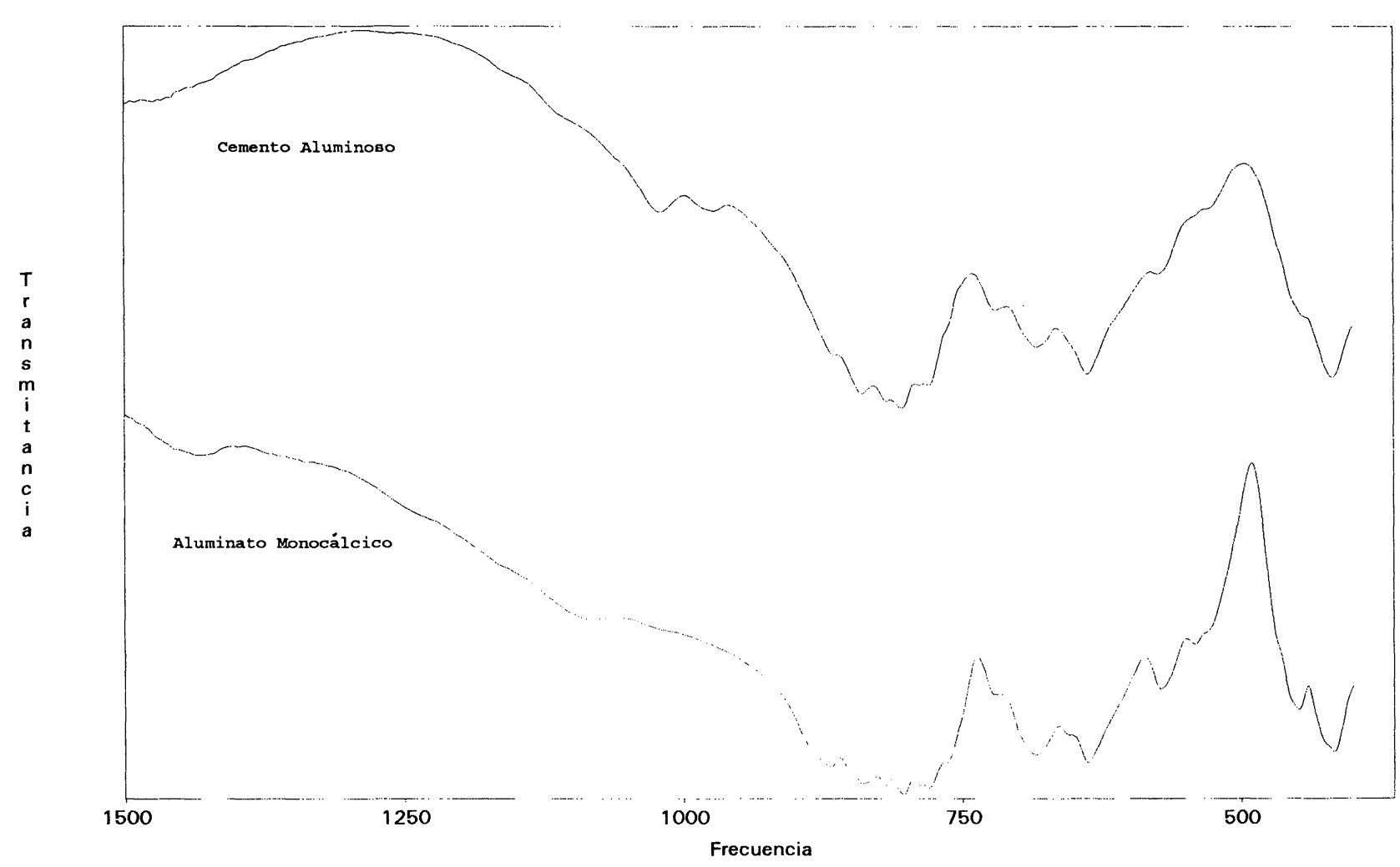

Fig. 6.- Espectros IR de un aluminato monocálcico y cemento aluminoso.

cálcico y de carbonatos cálcicos que no han sido detectados por difracción de rayos $\mathrm{X}$, lo que supone su pobre cristalinidad o bien que están en proporción inferior a la mínima detectable.

La zona inferior a $800 \mathrm{~cm}^{-1}$ es de gran complejidad. Destacan las bandas características del cemento aluminoso anhidro que aún permanece en la muestra.

- El espectro correspondiente a la muestra hidratada con agua caliente durante 7 días, es muy diferente al anterior. Entre 3.400 y $3.700 \mathrm{~cm}^{-1}$ presenta unas bandas que se interpretan como sigue:

* banda aguda e intensa en $3.663 \mathrm{~cm}^{-1}$ que corresponde al aluminato tricálcico hidratado, cúbico.

* Bandas intensas en 3.527 y $3.466 \mathrm{~cm}^{-1}$ y un "hombro" en 3.624 que corresponden a gibsita. Asimismo las bandas en 1.024 y $971 \mathrm{~cm}^{-1}$ son de este compuesto.

* En el resto del espectro IR las bandas de los aluminatos no son apropiadas para un adecuado diagnóstico, debido a la falta de simetría de las bandas Al-O que pueden dar lugar a una serie de vibraciones débilmente acopladas (3).

\subsection{Cemento aluminoso extraído de viguetas}

\subsubsection{Ejemplo I}

En la Fig. 8 se muestran los espectros de cuatro muestras extraídas de hormigón de cemento aluminoso de otras tantas viguetas que se encontraban ubicadas en distintos lugares en un mismo edificio. Se realizaron los difractogramas de rayos $\mathrm{X}$ y los análisis térmicos correspondientes. Se comprobó la presencia de gibsita y de bayerita en las muestras 1 y 4 . La muestra 2 tan sólo contenía gibsita. La muestra 3 presentaba líneas de difracción, muy poco intensas, de monocarboaluminato. La muestra 2 también tenía monocarboaluminato, en una proporción superior al de la muestra 3. La interpretación que se realiza de los espectros IR confirma y complementa todos estos resultados.

Todas las muestras han evolucionado en gran parte hasta su estado final, ya que no se observan bandas de aluminatos cálcicos hidratados; es decir, están en su totalidad carbonatadas, con la excepción de lo que en estos aspectos supone la presencia de los carboaluminatos, susceptibles aún de evolucionar. En todos los espectros aparecen las bandas de carbonatos y de hidróxidos de aluminio, especies químicas en diversas formas cristalinas. 


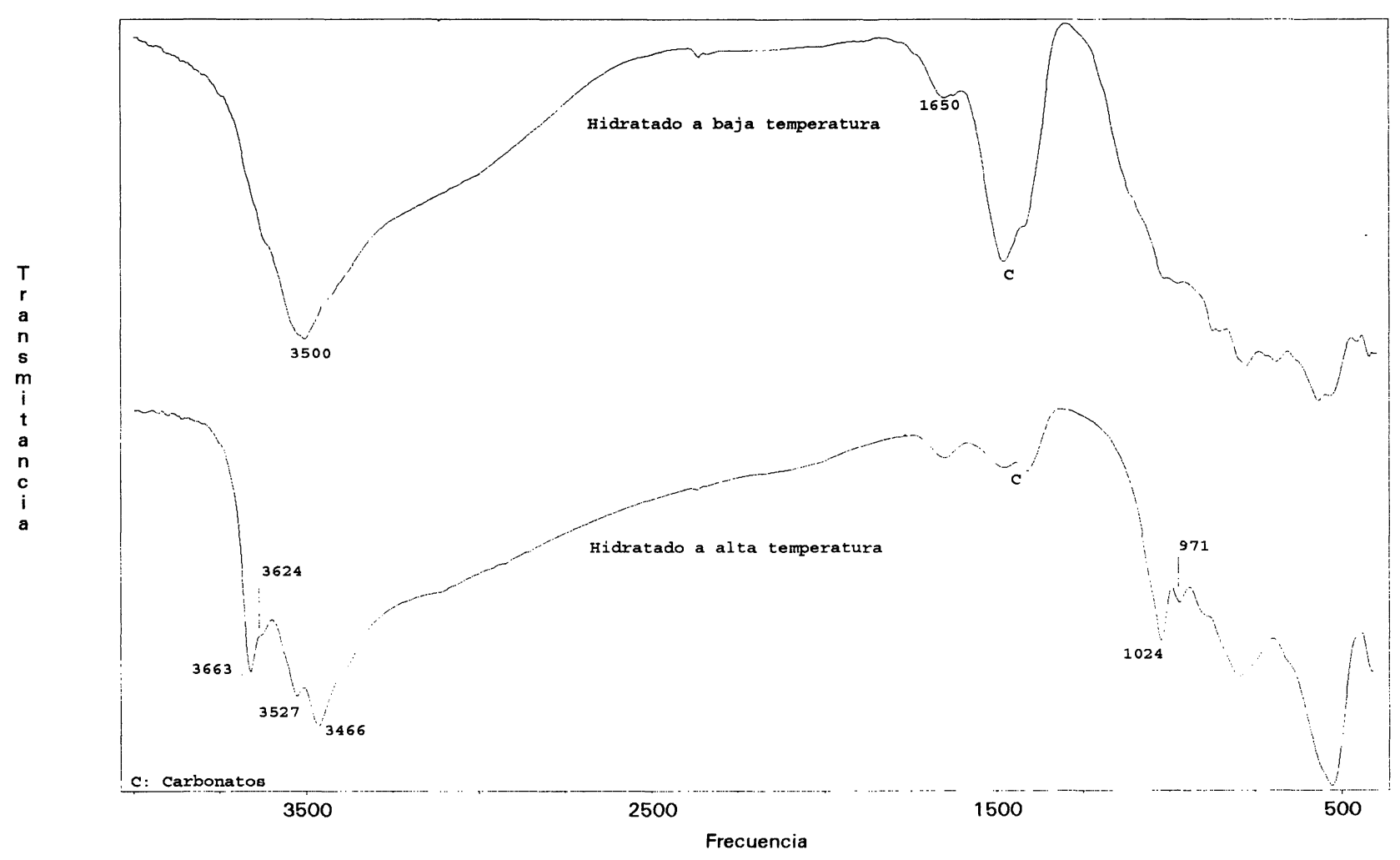

Fig. 7.- Espectros IR de cementos aluminosos hidratados a alta y baja temperatura.

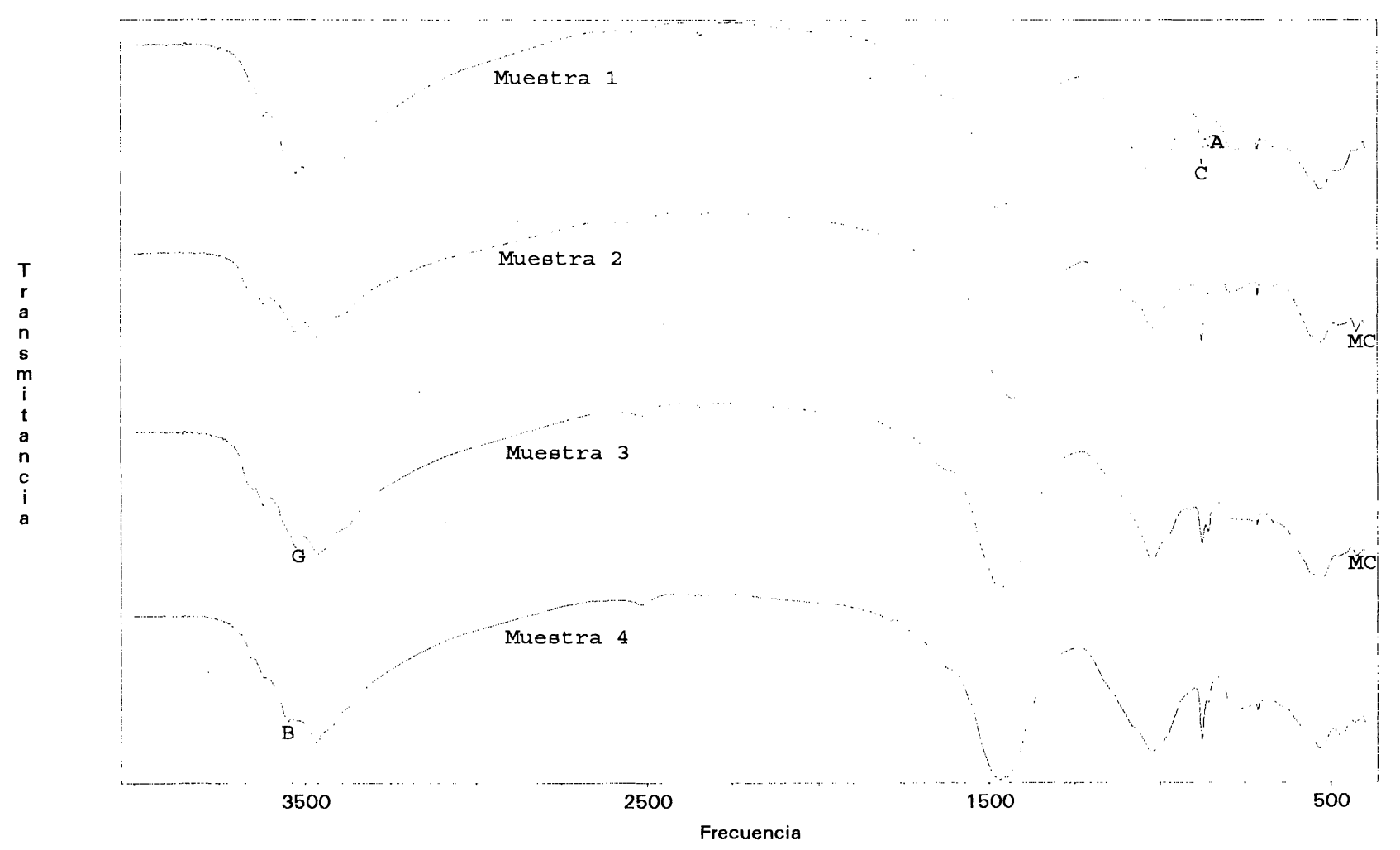

Fig. 8.- Espectros IR de las muestras 1. 2, 3 y 4 
En la Tabla IV se da un esquema orientativo acerca de las fases presentes en las muestras, la posición de algunas bandas clave así como la proporción relativa de estas fases en las muestras.

Las bandas características de los carbonatos cálcicos demuestran la presencia de calcita en todas las muestras y de aragonito (banda muy intensa hacia $1.485 \mathrm{~cm}^{-1} \mathrm{y}$ débil, definida, en $858 \mathrm{~cm}^{-1}$ ) en las muestras $2,4 \mathrm{y}$-en menor proporción- en la muestra 1 . La absorción más intensa de los monocarboaluminatos (1.350-1.400 $\left.\mathrm{cm}^{-1}\right)$ esta enmascarada por la banda muy fuerte de los carbonatos cálcicos. Por ello, la banda débil hacia $426 \mathrm{~cm}^{-1}$ es la más eficaz para la asignación del monocarboaluminato. Es interesante comprobar que la muestra 2 , que no contiene bayerita, es la única que no contiene aragonito.

\subsubsection{Ejemplo II}

Otro ejemplo del estudio de hormigón de cemento aluminoso a través de IR se da en la Fig. 9 en la que se exponen los espectros correspondientes a una fracción de cemento extraído de una vigueta deteriorada. En ésta se observaban dos zonas de la vigueta bien diferenciadas: la parte externa, un espesor aproximado de $3 \mathrm{~cm}$, presentaba una tonalidad pardo achocolatada; la zona interna, un color gris azulado. Por difracción de rayos $\mathrm{X}$ se demostró que la zona externa contenía gibsita y $\mathrm{CaCO}_{3}$ como aragonito y calcita. La zona interna contenía $\mathrm{C}_{3} \mathrm{AH}_{6}$, gibsita y menores proporciones de $\mathrm{CaCO}_{3}$. Había también cuarzo procedente de los áridos.

Laespectroscopía IR confirma plenamente esos resultados: en la muestra de la parte interna aparece claramente la banda aguda en $3.660 \mathrm{~cm}^{-1}$, asignada al aluminato tricálcico hidratado, y hacia 3.520 y $3.470 \mathrm{~cm}^{-1}$ las bandas de la gibsita. La banda muy intensa de cúbico enmascara a las finas de la gibsita. Hacia $1.370 \mathrm{~cm}^{-1}$ se observa con nitidez el amplio "hombro" asignado a monocarboaluminato. El doblete en $1.400-1.500 \mathrm{~cm}^{-1}$ es producido por la calcita y aragonito.

El espectro de la muestra exterior confirma el efecto de una profunda carbonatación que ha hecho desaparecer el aluminato tricálcico hidratado, el carboaluminato y aumentar notablemente la proporción de $\mathrm{CaCO}_{3}$ (calcita y aragonito). Probablemente haya algo de $\mathrm{Al}(\mathrm{OH})_{3}$ en forma gel, lo que influye en la alteración del espectro de la muestra en la zona $3.400-3.600 \mathrm{~cm}^{-1}$.

\section{AGRADECIMIENTO}

Los autores desean agradecer a laDIRECCIÓN GENERAL DE INVESTIGACIÓN CIENTÍFICA Y TÉCNICA, del MINISTERIO DE EDUCACION Y CIENCIA, por la subvención concedida a través del Programa Sectorial de Promoción General del Conocimiento (PB92-0100), sin la cual no hubiera podido realizarse el presente trabajo.

TABLA IV

\begin{tabular}{|c|c|c|c|c|c|}
\hline & $\begin{array}{l}\text { Frecuencia } \\
\qquad\left(\mathrm{cm}^{-1}\right)\end{array}$ & Muestra 1 & Muestra 2 & Muestra 3 & Muestra 4 \\
\hline \multirow{2}{*}{ Bayerita } & 3.550 & ++ & -- & + & +++ \\
\hline & 3.430 & si & no & si & si \\
\hline Gibsita & 3.526 & ++ & +++ & ++ & + \\
\hline Monocarb. & 420 & -- & ++ & + & -- \\
\hline Aragonito & 1.485 & ++ & -- & ++ & + \\
\hline \multirow{2}{*}{ Calcita } & 1.423 & +++ & +++ & +++ & +++ \\
\hline & 858 & +++ & -- & +++ & +++ \\
\hline
\end{tabular}

+++ muy abundante, ++ abundante, + poco. 


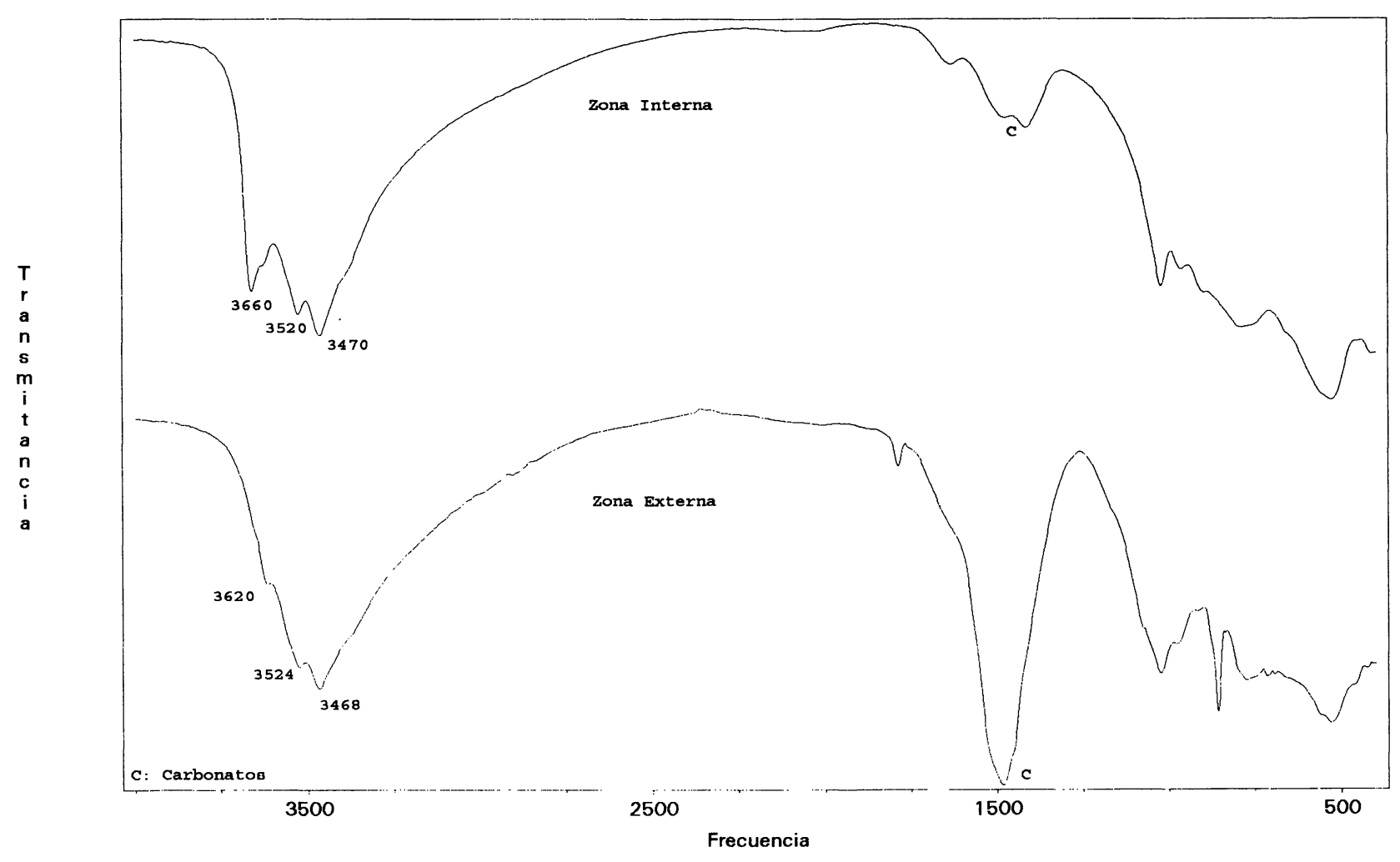

Fig. 9.- Espectros IR de unas muestras reales de cemento aluminoso.

\section{BIBLIOGRAFÍA}

(1) VÁZQUEZ, T., TRIVIÑo, F.y RUIZ DE GAUNA, A.: Monografía del IETCC nº 334 . Madrid, julio de 1976.

(2) VOLANT, J.: Thèse de Doctorat. Fac. des Sciences de l'Université de Paris. Revue des Mat. des Const. $\mathbf{n}^{\mathrm{a}}$ 632, pp. 191-197; $\mathrm{n}^{\circ}$ 633-634, pp. 257-272; n635-636, pp. 313-323; n638, pp. 432-450. (1968).

(3) BENSTED J.: Tonind.-Ztg., n $^{\circ} 10$. pp. 365-366, año 1976.

(4) ROBERTS, M.H.: J. appl. Chem., pp. 543-546, oct. 1957.

(5) BENSTED, J.: World Cement Technology. p. 85, marzo, 1982.

(6) BARNISHEL, R.I y RICH, C.I.: Proc. Soil. Sci. Soc. Amer., n²9, pp. 531-539, año 1965.

(7) HSU, P. H.: Soil Sci. Soc. Amer. Proc. n 30, pp. 173-176, año 1966.

(8) PUERTAS, F., FERNÁNDEZ-CARRASCO, L., BLANCO-VARELA, T., VÁZQUEZ, T. y DE LA FUENTE, A.: Primer Congreso. El csd dels sostres. Experiencies i perspectivas. Comunicación Científica $\mathbf{n}^{\circ} 26$. Barcelona, 1995.

(9) VAN DER MAREL y BEUTELSPACHER, H.: Atlas of Infrared Spectroscopy of Clay Minerals and Their Admixtures. Ed. ELSEVIER. New YorK. 1976.

(10) FREDERICKSON, L.D.: Analytcal Chemistry. vol. 26, p. 1883, dic. 1954.

(11) MOENKE, HORST.: Mineralspectren. Akademie-Verlag-Berlín. 1962.

(12) SATO, TAICHI.: J. appl. Chem., pp. 207-209. año 1961. 